\title{
Performance Optimization for UAV-Enabled Wireless Communications under Flight Time Constraints
}

\author{
Mohammad Mozaffari ${ }^{1}$, Walid Saad ${ }^{1}$, Mehdi Bennis ${ }^{2}$, and Mérouane Debbah ${ }^{3}$ \\ ${ }^{1}$ Wireless@VT, Electrical and Computer Engineering Department, Virginia Tech, VA, USA, \\ Emails:\{mmozaff,walids\}@vt.edu. \\ ${ }^{2}$ CWC - Centre for Wireless Communications, Oulu, Finland, Email: bennis@ee.oulu.fi. \\ ${ }^{3}$ Mathematical and Algorithmic Sciences Lab, Huawei France R \& D, Paris, France, and CentraleSupelec, \\ Universite Paris-Saclay, Gif-sur-Yvette, France, Email: merouane.debbah@huawei.com.
}

\begin{abstract}
In this paper, the effective use of unmanned aerial vehicles (UAVs) as flying base stations that can provide wireless service to ground users is investigated. In particular, a novel framework for optimizing the performance of such UAV-based wireless systems, in terms of the average number of bits (data service) transmitted to users under flight time constraints, is proposed. In the considered model, UAVs are deployed over a given geographical area to serve ground users that are distributed within a given area based on an arbitrary spatial distribution function. In this case, based on the maximum possible flight times of the UAVs, the average data service delivered to the users is maximized by finding the optimal cell partitions associated to the UAVs, under a fair resource allocation scheme. To this end, using the powerful mathematical framework of optimal transport theory, a gradient-based algorithm is proposed for optimally partitioning the geographical area based on the users' distribution, flight times, and locations of the UAVs. Simulation results show that the proposed cell partitioning approach yields a significantly higher fairness among the users compared to the classical weighted Voronoi diagram. In particular, by using our approach, the Jain's fairness index is improved by a factor of 2.6.
\end{abstract}

\section{INTRODUCTION}

Recently, the use of aerial platforms such as unmanned aerial vehicles (UAVs), drones, and balloons has emerged as a promising solution for providing reliable and cost-effective wireless communication services for ground wireless devices [1]-[3]. In particular, UAVs can be deployed as flying base stations to provide coverage expansion and capacity enhancement of terrestrial cellular networks [1], [3]-[5]. With their inherent attributes such as mobility, flexibility, and adaptive altitude, UAVs have several key potential applications in wireless systems. For instance, UAVs can be deployed to complement existing cellular systems by providing additional capacity to hotspot areas during temporary events. In addition, UAV-based wireless communications can provide fast, reliable and cost-effective connectivity to areas that are poorly covered by terrestrial networks. For instance, Google Loon and Facebook's Internet-delivery drone projects aim to provide a global wireless connectivity by employing such aerial platforms [4]. To reap the benefits of using UAVs as aerial base stations, one must address many technical challenges such as deploy-

This research was supported by the U.S. National Science Foundation under Grants AST-1506297 and ACI-1541105, by the U.S. Office of Naval Research (ONR) under Grant N00014-15-1-2709, and, by the ERC Starting Grant 305123 MORE (Advanced Mathematical Tools for Complex Network Engineering). ment, air-to-ground channel modeling, user association, and flight time optimization [5].

For instance, in [4], the authors studied the efficient deployment of aerial base stations to maximize the coverage and rate performance of wireless networks. The work in [6] performed air-to-ground channel modeling for UAV-based communications. In [7], the authors introduced a framework for trajectory optimization and energy-efficient UAV-based communication. The work in [8] studied the joint optimization of user scheduling and UAV trajectory to maximize the minimum rate of users.

Another important challenge in UAV-based communications is user (or cell) association. The work in [9] investigated the area-to-UAV assignment for capacity enhancement of heterogeneous wireless networks. However, this work is limited to the case with a uniform spatial distribution of ground users, and it ignores the UAVs' flight time constraints. The work in [10] derived the delay-optimal cell association policy in a UAV-enabled cellular network. However, in [10], the impact of flight time constraints on the system performance is not taken into account. Indeed, the flight time duration of the UAVs presents a unique design challenge for UAV-based communication systems [11]. In particular, the performance of such systems significantly depends on the flight duration that each UAV can dedicate to provide wireless service to ground users. Hence, while analyzing UAV-based communication systems, the flight time constraints must be also taken into account. In consequence, there is a need for a new framework that can be used to analyze and optimize the performance of UAVbased communications under flight time constraints. To our best knowledge, none of the previous UAV studies such as [1][10], considered the flight time constraints in their analysis.

The main contribution of this paper is a novel framework for optimized UAV-to-ground communications under explicit UAVs' flight time constraints. In particular, we consider a network in which multiple UAVs are deployed as aerial base stations to provide wireless service to ground users that are distributed over a geographical area based on an arbitrary spatial distribution. In the considered model, given the maximum possible flight time of UAVs, that is imposed by the limited on-board energy of UAVs and flight regulations, we maximize the average number of bits (data service) transmitted to the users under a fair resource allocation scheme. To this end, based on the flight times and the spatial distribution 
of users, we find the optimal cell partitions associated to the UAVs. Using the powerful mathematical framework of optimal transport theory [12], we propose a gradient-based algorithm that optimally partitions the geographical area based on the users' distribution as well as the UAVs' flight times and locations. The results show that our proposed cell partitioning approach, under the flight time considerations, leads to a significantly higher fairness among the users compared to the classical weighted Voronoi approach. In particular, the Jain's fairness index is improved by a factor of 2.6.

The rest of this paper is organized as follows. In Section II, we present the system model. Our cell partitioning problem is presented in Section III. In Section IV we detail our approach to find the optimal cell partitions. Simulation results are provided in Section V and conclusions are drawn in Section VI.

\section{SySTEM MODEL}

Consider a geographical area $\mathcal{D} \subset \mathbb{R}^{2}$ within which a number of wireless users are located according to a given distribution $f(x, y)$ in the two-dimensional plane. In this area, a set $\mathcal{M}$ of $M$ UAVs are used as aerial base stations to provide wireless service for the ground users ${ }^{1}$. Let $s_{i}=\left(x_{i}, y_{i}, h_{i}\right)$ be the three-dimensional coordinate of each UAV $i \in \mathcal{M}$ with $h_{i}$ being the altitude of UAV $i$. We consider a downlink scenario in which each UAV adopts a frequency division multiple access (FDMA) technique to provide service for the ground users. Let $P_{i}$ and $B_{i}$ be, respectively, the maximum transmit power and the total available bandwidth for UAV $i$. Moreover, we use $A_{i}$, as shown in Fig. 1, to denote the partition of the geographical area which is served by UAV $i$. In this case, all users located in cell partition $A_{i}$ will be connected to UAV $i$. Hence, the geographical area is divided into $M$ disjoint partitions each of which is associated with one of the UAVs. Let $\tau_{i}$ be the flight time duration of UAV $i$ which is used to provide wireless service to ground users. During the flight time, the UAV must initiate connections to the ground users, perform required computations, and transmit data to the users. Let $T_{i}$ be the effective data transmission period during which a UAV services the users. In general, the effective data transmission time is less than the total flight time. Consequently, we consider a control time as $g_{i}($.$) ,$ a function of the number of users in $\mathcal{A}_{i}$, to represent the portion of the flight time that is not used for the effective data transmission. This control time naturally captures the total time that a UAV $i$ needs to spend for computations, setting up connections, and control signaling.

In our model, we use the term data service to represent the amount of data (in bits) that each UAV transmits to a given user. Clearly, the data service depends on several factors such as the effective data transmission time (which is directly related to flight time) and the transmission bandwidth. Therefore, the effective data transmission times and the bandwidth of the UAVs are considered as the resources needed to service the users. Given this model, we analyze UAV-based

\footnotetext{
${ }^{1}$ For wireless backhauling of aerial networks, satellite and WiFi are considered as the two feasible candidates.
}

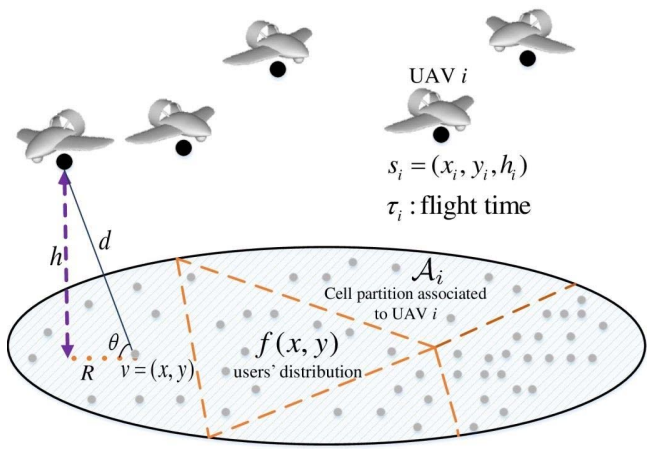

Fig. 1: System model.

communications under flight time constraints. In particular, given the maximum possible flight times (imposed by the energy and flight limitations of each UAV), we maximize the average data service to the users under a fair resource allocation policy by optimal cell partitioning of the area. Here, we optimally partition the geographical area based on the flight times and the spatial distribution of users. As a result, given the maximum possible flight time of each UAV, the total data service under user fairness considerations is maximized. Next, we present the air-to-ground path loss model for UAV-user communications which is used to perform our analysis.

The air-to-ground signal propagation is affected by the obstacles and buildings in the environment. Depending on the propagation environment, air-to-ground communication links can be either LoS or non-line-of-sight (NLoS). Clearly, the probability of having LoS communication links depends on the locations, heights, and the number of obstacles, as well as the elevation angle between a given UAV and it's served ground user. In our model, we consider a widely used probabilistic path loss model provided by International Telecommunication Union (ITU-R), and the work in [6]. The path loss between UAV $i$ and a given user at location $(x, y)$ can be given by [6]: $\Lambda_{i}(x, y)=\left\{\begin{array}{l}\left(\frac{4 \pi f_{c} d_{o}}{c}\right)^{2}\left(d_{i}(x, y) / d_{o}\right)^{2} \mu_{\mathrm{LoS}}, \quad \text { LoS link, } \\ \left(\frac{4 \pi f_{c} d_{o}}{c}\right)^{2}\left(d_{i}(x, y) / d_{o}\right)^{2} \mu_{\mathrm{NLoS}}, \quad \text { NLoS link, }\end{array}\right.$

where $\mu_{\mathrm{LoS}}$ and $\mu_{\mathrm{NLoS}}$ are different attenuation factors considered for LoS and NLoS links. $f_{c}$ is the carrier frequency, $c$ is the speed of light, and $d_{o}$ is the free-space reference distance. Also, $d_{i}(x, y)=\sqrt{\left(x-x_{i}\right)^{2}+\left(y-y_{i}\right)^{2}+h_{i}^{2}}$ is the distance between UAV $i$ and an arbitrary ground user located at $(x, y)$. For the UAV-user link, the LoS probability is given by [6]:

$$
P_{\mathrm{LoS}, i}=b_{1}\left(\frac{180}{\pi} \theta_{i}-15\right)^{b_{2}},
$$

where $\theta_{i}=\sin ^{-1}\left(\frac{h_{i}}{d_{i}(x, y)}\right)$ is the elevation angle (in radians) between the UAV and the user. Also, $b_{1}$ and $b_{2}$ are constant values reflecting the environment impact. Note that, the NLoS probability is $P_{\mathrm{NLoS}, i}=1-P_{\mathrm{LoS}, i}$. Clearly, considering $d_{o}=$ $1 \mathrm{~m}$, and $K_{o}=\left(\frac{4 \pi f_{c}}{c}\right)^{2}$ the average path loss is

$K_{o} d_{i}{ }^{2}(x, y)\left[P_{\mathrm{LoS}, i} \mu_{\mathrm{LoS}}+P_{\mathrm{NLoS}, i} \mu_{\mathrm{NLoS}}\right]$. Hence, the received signal power from UAV $i$ will be:

$$
\bar{P}_{r, i}(x, y)=\frac{P_{i}}{K_{o} d_{i}{ }^{2}(x, y)\left[P_{\mathrm{LoS}, i} \mu_{\mathrm{LoS}}+P_{\mathrm{NLoS}, i} \mu_{\mathrm{NLoS}}\right]},
$$


where $P_{i}$ is the UAV's transmit power. Then, the received SINR for a user located at coordinates $(x, y)$ while connecting to $\mathrm{UAV} i$ will be:

$$
\gamma_{i}(x, y)=\frac{\bar{P}_{r, i}(x, y)}{I_{i}(x, y)+\sigma^{2}},
$$

where $I_{i}(x, y)=\beta \sum_{j \neq i} \bar{P}_{r, j}(x, y)$ is the received interference at location $(x, y)$ stemming from all UAVs except UAV $i$. We also consider a weight factor $0 \leq \beta \leq 1$ to adjust the amount of interference and capture the impact of any interference mitigation technique. Naturally, $\beta=1$ and $\beta=0$ correspond to the full interference and interference-free scenarios.

Clearly, the throughput of a user located at $(x, y)$ if it connects to UAV $i$ is:

$$
C_{i}(x, y)=W(x, y) \log _{2}\left(1+\gamma_{i}(x, y)\right),
$$

where $W(x, y)$ is the bandwidth allocated to the user at $(x, y)$.

Subsequently, the total data service for the user provided by the UAV will be:

$$
L_{i}(x, y)=T_{i} C_{i}(x, y),
$$

where $T_{i}$ is the effective transmission time of UAV $i$. Also, $L_{i}(x, y)$ represents the total number of bits transmitted to the user located at $(x, y)$. Note that, the data service offered to each ground user depends on a number of key parameters such as the location of the user and the serving UAV, the bandwidth allocated to the user, and the effective data transmission time of the UAV. Here, the resources used by the UAVs to service the ground users are the bandwidth and the effective data transmission times. Clearly, the amount of resources that each user can receive depends on the total number of users, cell partitions as well as bandwidth and flight times of the UAVs. Next, we present our optimal cell partitioning problem.

\section{iII. Cell Partitioning Problem for Data Service MAXIMIZATION UNDER FAIR RESOURCE ALLOCATION}

In this section, we present our cell partitioning problem whose goal is to maximize the average data service based on the UAVs' flight times as well as the spatial distribution of users. Here, each cell partition is assigned to one UAV, and the users within the cell partition must be serviced by the corresponding UAV. We note that, in classical cell partitioning approaches such as Voronoi and weighted Voronoi diagrams, the spatial distribution of users is not taken into account. As a result, some partitions can be highly congested with users and, hence, each user will receive significantly lower amount of resources than those in less congested partitions. Thus, such classical cell partitioning approaches can lead to a highly unfair data service for the users. In our cell partitioning problem, however, while maximizing the total data service, we ensure that the resources are equally shared among the users. Hence, our approach avoids creating unbalanced cell partitions and, thus, it leads to a higher level of fairness compared to the Voronoi case.

Let $\tau_{i}$ be the flight time of UAV $i$ over its cell partition $\mathcal{A}_{i}$. The flight time is composed of the effective data transmission time and the control time. To ensure a fair resource allocation, we consider the following fairness criterion:

$$
T_{i}=\tau_{i}-g_{i}\left(\int_{\mathcal{A}_{i}} f(x, y) \mathrm{d} x \mathrm{~d} y\right), \forall i \in \mathcal{M},
$$

where $g_{i}$ is the control time which depends on the number of the users located in $\mathcal{A}_{i}$. Note also that, given the spatial distribution of users, $f(x, y)$, and the total number of users, $N$, the average number of users in partition $\mathcal{A}_{i}$ is equal to $N \int_{\mathcal{A}_{i}} f(x, y) \mathrm{d} x \mathrm{~d} y$. From (5) and (6), which are used to compute the amount of data service, we can see that the value $T_{i} B_{i}$ can be considered as the resources that UAV $i$ uses to service users in $\mathcal{A}_{i}$. In this case, under a fair resource allocation policy, we should have:

$$
\frac{T_{i} B_{i}}{\int_{\mathcal{A}_{i}} f(x, y) \mathrm{d} x \mathrm{~d} y}=\frac{T_{j} B_{j}}{\int_{\mathcal{A}_{j}} f(x, y) \mathrm{d} x \mathrm{~d} y}, \quad \forall i \neq j \in \mathcal{M},{ }^{2}
$$

where (8) ensures that a UAV with more resources (bandwidth and flight time) will serve a higher number of users.

Using (8) and considering the fact that $\int_{\mathcal{D}} f(x, y) \mathrm{d} x \mathrm{~d} y=$ $\sum_{k=1}^{M} \int_{\mathcal{A}_{k}} f(x, y) \mathrm{d} x \mathrm{~d} y=1$, we have the following constraint on the number of users in each partition:

$$
\int_{\mathcal{A}_{i}} f(x, y) \mathrm{d} x \mathrm{~d} y=\frac{B_{i} T_{i}}{\sum_{k=1}^{M} B_{k} T_{k}}, \forall i \in \mathcal{M} .
$$

As we can see from (9), the number of users in each generated optimal partition will depend on the UAVs' resources. Clearly, when the UAVs have the same flight times and bandwidths, (7)-(9) lead to $\int_{\mathcal{A}_{i}} f(x, y) \mathrm{d} x \mathrm{~d} y=\frac{1}{M}, \forall i \in \mathcal{M}$. This case implies that identical UAVs will service equally-loaded cell partitions. Given (5), (6), and (9), we can write the average data service at location $(x, y) \in \mathcal{A}_{i}$ as:

$$
\begin{aligned}
L_{i}(x, y) & =\frac{T_{i} B_{i}}{N \int_{\mathcal{A}_{i}} f(x, y) \mathrm{d} x \mathrm{~d} y} \log _{2}\left(1+\gamma_{i}(x, y)\right) \\
& =\left(\frac{1}{N} \sum_{k=1}^{M} B_{k} T_{k}\right) \log _{2}\left(1+\gamma_{i}(x, y)\right) .
\end{aligned}
$$

Now, we formulate an optimization problem that seeks to maximize the average data service by optimal partitioning of the target area. The data service maximization problem is given by:

$$
\begin{aligned}
& \max _{\mathcal{A}_{i}, i \in \mathcal{M}} \sum_{i=1}^{M} \int_{\mathcal{A}_{i}} L_{i}(x, y) f(x, y) \mathrm{d} x \mathrm{~d} y, \\
& \text { s.t. } \int_{\mathcal{A}_{i}} f(x, y) \mathrm{d} x \mathrm{~d} y=\frac{B_{i} T_{i}}{\sum_{k=1}^{M} B_{k} T_{k}}, \forall i \in \mathcal{M}, \\
& \gamma_{i}(x, y) \geq \gamma_{t h}, \quad \text { if }(x, y) \in \mathcal{A}_{i}, \quad \forall i \in \mathcal{M}, \\
& \mathcal{A}_{l} \cap \mathcal{A}_{m}=\emptyset, \quad \forall l \neq m \in \mathcal{M}, \quad \bigcup_{i \in \mathcal{M}} \mathcal{A}_{i}=\mathcal{D},
\end{aligned}
$$

where (12) captures the constraint on the load of each cell partition. Also, (13) is the necessary condition for connecting

\footnotetext{
${ }^{2}$ Note that, given flight times of the UAVs, $\tau_{i}, \forall i \in \mathcal{M}$, we can compute $T_{i}, \forall i \in \mathcal{M}$ by solving the system of equations in (7) and (8).
} 
each user to a UAV $i$. (14) ensures that the cell partitions are disjoint and their union covers the entire target area $\mathcal{D}$.

Considering the constraint in (13), we define the function $q_{i}(x, y)=\left(\frac{\gamma_{i}(x, y)}{\gamma_{\mathrm{th}}}\right)^{-n}$ with $n$ being a large number (i.e. tends to $+\infty)$, and, then, we subtract $q_{i}(x, y)$ from the objective function in (11). Clearly, when (13) is violated, $q_{i}(x, y)$ tends to $+\infty$ and, hence, point $(x, y)$ will not be assigned to UAV $i$ or equivalently $(x, y) \notin \mathcal{A}_{i}$. Therefore, assuming that the problem is feasible, we can remove (13) while penalizing the objective function in (11) by $q_{i}(x, y)$. Now, by defining $\omega_{i}=$ $\frac{B_{i} T_{i}}{\sum_{k=1}^{M} B_{k} T_{k}}$, the maximization problem in (11) can be rewritten as the following minimization problem:

$$
\begin{aligned}
& \min _{\mathcal{A}_{i}, i \in \mathcal{M}} \sum_{i=1}^{M} \int_{\mathcal{A}_{i}}-\left(L_{i}(x, y)-q_{i}(x, y)\right) f(x, y) \mathrm{d} x \mathrm{~d} y, \\
& \text { s.t. } \quad \int_{\mathcal{A}_{i}} f(x, y) \mathrm{d} x \mathrm{~d} y=\omega_{i}, \quad \forall i \in \mathcal{M}, \\
& \quad \mathcal{A}_{l} \cap \mathcal{A}_{m}=\emptyset, \quad \forall l \neq m \in \mathcal{M}, \quad \bigcup_{i \in \mathcal{M}} \mathcal{A}_{i}=\mathcal{D} .
\end{aligned}
$$

Solving the optimization problem in (15) is challenging due to various reasons. First, the optimization variables $\mathcal{A}_{i}$, $\forall i \in \mathcal{M}$, are sets of continuous partitions (as we have a continuous area) which are mutually dependent. Second, to perfectly capture the spatial distribution of users, $f(x, y)$ is considered to be a generic function of $x$ and $y$ and, this leads to the complexity of the given two-fold integrations. In addition, due to the constraints given in (16), finding $\mathcal{A}_{i}$ becomes more challenging. To solve the optimization problem in (15), we model the problem by exploiting optimal transport theory [12].

\section{Optimal Transport Theory for Optimal Cell PARTITIONING}

Optimal transport theory was initiated by the Monge's problem which is stated as follows [12]. Given piles of sands and holes with the same volume, what is the best move (transport map) to entirely fill up the holes with the minimum total transportation cost. Mathematically, the Monge optimal transport problem can be written as follows. Given two probability distributions $f_{1}$ on $X \subset \mathbb{R}^{n}$, and $f_{2}$ on $Y \subset \mathbb{R}^{n}$, find the optimal transport map $G$ from $f_{1}$ to $f_{2}$ that minimizes the following problem:

$$
\min _{G} \int_{X} c(\boldsymbol{x}, G(\boldsymbol{x})) f_{1}(\boldsymbol{x}) \mathrm{d} \boldsymbol{x} ; G: X \rightarrow Y,
$$

where $c(\boldsymbol{x}, G(\boldsymbol{x}))$ denotes the cost of transporting a unit mass from a location $\boldsymbol{x} \in X$ to a location $\boldsymbol{y}=G(\boldsymbol{x}) \in Y$.

Given this optimal transport framework, we can solve our optimization problem in (15). In particular, we model this problem as a semi-discrete optimal transport problem in which the source measure (users' distribution) is continuous while the destination (UAVs' distribution) is discrete. Using optimal transport theory, we can find the optimal cell partitions, $\mathcal{A}_{i}$, for which the average total data service is maximized. In our model, users have a continuous distribution, and the locations of the UAVs can be considered as discrete points. Then, the optimal cell partitions are obtained by optimally mapping the users to the UAVs. In fact, given (15), the cell partitions are related to the transport map by [13]:

$$
\left\{G(\boldsymbol{v})=\sum_{i \in \mathcal{M}} \boldsymbol{s}_{i} \mathbb{1}_{\mathcal{A}_{i}}(\boldsymbol{v}) ; \int_{\mathcal{A}_{i}} f(x, y) \mathrm{d} x \mathrm{~d} y=\omega_{i}\right\},
$$

where $\omega_{i}=\frac{B_{i} T_{i}}{\sum_{k=1}^{M} B_{k} T_{k}}$, as given in (16), is directly related to the flight time and the bandwidth of the UAVs. $\mathbb{1}_{\mathcal{A}_{i}}(\boldsymbol{v})$ is the indicator function which is 1 if $v \in \mathcal{A}_{i}$, and 0 otherwise.

Therefore, the optimization problem in (15) can be cast within the optimal transport framework as follows. Given a continuous probability measure $f$ of users, and a discrete probability measure $\Gamma=\sum_{i \in \mathcal{M}} \omega_{i} \delta_{\boldsymbol{s}_{i}}$ corresponding to the UAVs, we must find the optimal transport map for which $\int_{\mathcal{D}} J(\boldsymbol{v}, G(\boldsymbol{v})) f(x, y) \mathrm{d} x \mathrm{~d} y$ is minimized. In this case, $\delta_{\boldsymbol{s}_{i}}$ is the Dirac function, and $J$ is the transportation cost function which is used in (15) and is given by:

$$
J\left(\boldsymbol{v}, \boldsymbol{s}_{i}\right)=J\left(x, y, \boldsymbol{s}_{i}\right)=q_{i}(x, y)-L_{i}(x, y) .
$$

Now, using the Kantorovich duality theorem [12] from optimal transport theory, we can transform (15) to the following optimization problem [13]:

$$
\max _{\psi_{i}, i \in M}\left\{F\left(\boldsymbol{\psi}^{T}\right)=\sum_{i=1}^{M} \psi_{i} \omega_{i}+\int_{D} \psi^{c}(x, y) f(x, y) \mathrm{d} x \mathrm{~d} y\right\},
$$

where $\boldsymbol{\psi}^{T}$ is a vector of variables $\psi_{i}, \forall i \in M$, and $\psi^{c}(x, y)=$ $\inf J\left(x, y, s_{i}\right)-\psi_{i}$. As a result, the optimization problem in $(15)$ is reduced to (21) with a set of $M$ optimization variables, $\psi_{i}, \forall i \in \mathcal{M}$. Given (21), we can further proceed to solve (15) by presenting the following theorem:

Theorem 1. Given (21), $F$ is a concave function of variables $\psi_{i}, i \in \mathcal{M}$. Also, we have:

$$
\frac{\partial F}{\partial \psi_{i}}=\omega_{i}-\int_{\mathcal{D}_{i}} f(x, y) \mathrm{d} x \mathrm{~d} y,
$$

where $\mathcal{D}_{i}=\left\{(x, y) \mid J\left(x, y, \boldsymbol{s}_{i}\right)-\psi_{i} \leq J\left(x, y, \boldsymbol{s}_{j}\right)-\psi_{j}, \forall j \neq i\right\}$.

Proof: Clearly, $\sum_{i=1}^{M} \psi_{i} \omega_{i}$ is a linear function of $\psi_{i}$. Also, given any $i \in \mathcal{M}, J\left(x, y, \boldsymbol{s}_{i}\right)-\psi_{i}$ is a linear function of $\psi_{i}$. Let $z\left(\boldsymbol{\psi}^{T}\right)=\inf J\left(x, y, \boldsymbol{s}_{i}\right)-\psi_{i}$ with $\boldsymbol{\psi}^{T}$ being a vector of all variables $\psi_{i}, i \in \mathcal{M}$. Then, we can observe that the hypograph of $z\left(\boldsymbol{\psi}^{T}\right)$, a set of points below $z\left(\boldsymbol{\psi}^{T}\right)$, is a convex set. Subsequently, considering the fact that a function is concave if and only if its hypograph is convex, we prove the concavity of $z\left(\boldsymbol{\psi}^{T}\right)$. Finally, since multiplying $z\left(\boldsymbol{\psi}^{T}\right)$ by a positive probability density function $f(x, y)$, and taking integration over $(x, y)$ does not violate the concavity, $F$ is also a concave function of $\boldsymbol{\psi}^{T}$. Now, to find the derivative of $F$ with respect to $\psi_{i}$, we first compute $\frac{\partial \psi^{c}}{\partial \psi_{i}}$, which is given by:

$\frac{\partial \psi^{c}}{\partial \psi_{i}}= \begin{cases}-1, & \text { if } J\left(x, y, \boldsymbol{s}_{i}\right)-\psi_{i} \leq J\left(x, y, \boldsymbol{s}_{j}\right)-\psi_{j}, i \neq j, \\ 0, & \text { otherwise. }\end{cases}$

Then, by defining

$$
\mathcal{D}_{i}=\left\{(x, y) \mid J\left(x, y, \boldsymbol{s}_{i}\right)-\psi_{i} \leq J\left(x, y, \boldsymbol{s}_{j}\right)-\psi_{j}, \forall j \neq i\right\},
$$




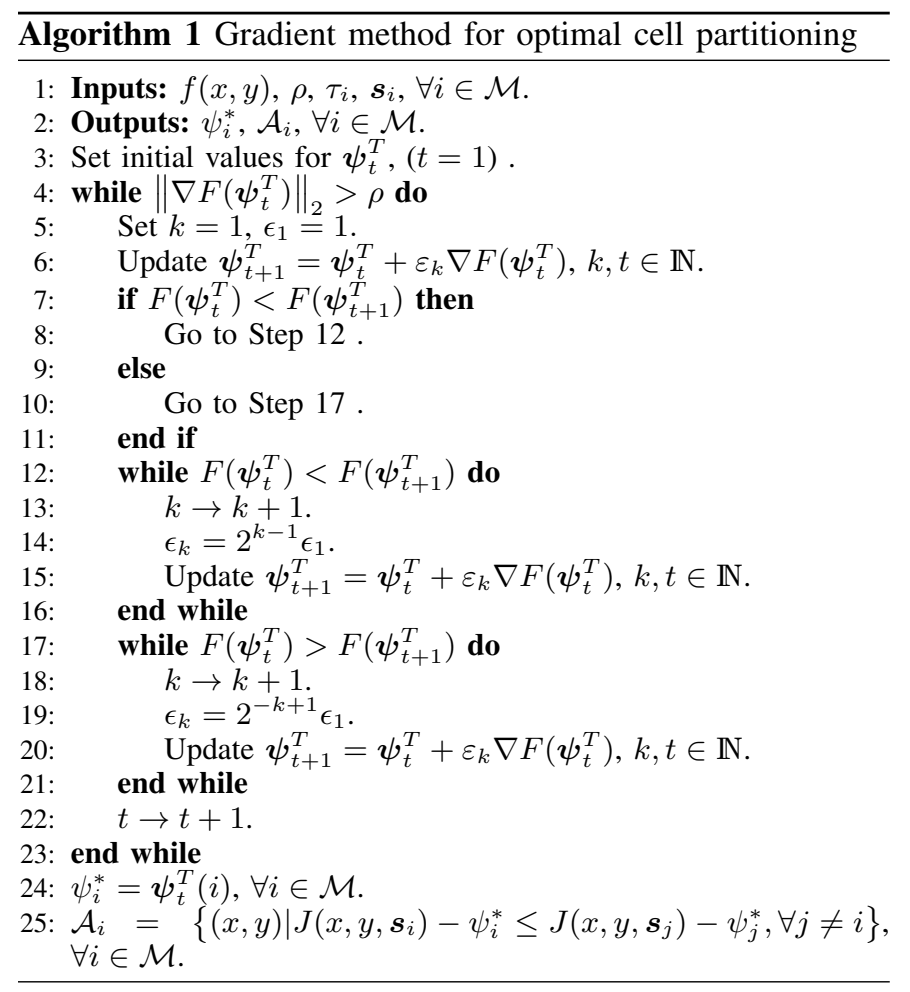

the derivative of $F$, given in (21), will be:

$$
\frac{\partial F}{\partial \psi_{i}}=\omega_{i}-\int_{\mathcal{D}_{i}} f(x, y) \mathrm{d} x \mathrm{~d} y .
$$

This proves the theorem.

Theorem 1 shows the concavity of $F$ as a function of $\boldsymbol{\psi}^{T}$. Thus, the optimal values for variables $\psi_{i}, \forall i \in \mathcal{M}$, can be obtained by maximizing $F$. Then, given the optimal $\psi_{i}, \forall i \in \mathcal{M}$, equation (19) is used to determine the optimal cell partitions corresponding to the optimization problem in (15). In this case, using the first derivative of $F$ provided in (22), we propose a gradient-based method to determine the optimal vector $\boldsymbol{\psi}^{T}$ that leads to the optimal cell partitions. Here, using the gradient descent method is simple in terms of implementation and does not require computing the Hessian matrix of $F$ which is needed in the Newton methods.

The proposed algorithm for finding the optimal cell partitions is shown as Algorithm 1 and proceeds as follows. The inputs are the distribution of users, flight times, locations of the UAVs, and $\rho>0$ which is the threshold based on which the algorithm stops. In Algorithm 1, we first initialize vector $\boldsymbol{\psi}_{t}^{T}$ with $t$ being the iteration number. Next, using (22), we compute $\nabla F\left(\boldsymbol{\psi}_{t}^{T}\right)$. In Step 6, we update $\boldsymbol{\psi}_{t}^{T}$ using step size $\epsilon_{k}$. The appropriate step size at each iteration is determined through Steps 7 to 21. In this case, the algorithm stops whenever condition in 4 is not satisfied. Clearly, due to the concavity of $F$, the optimal solution of (21) is attained. Finally, based on the optimal vector $\psi^{T}$, the optimal cell partitions are determined using Steps 24 and 25.

\section{Simulation Results and Analysis}

For our simulations, we consider a rectangular area of size $1000 \mathrm{~m} \times 1000 \mathrm{~m}$ in which the ground users are distributed
Table I: Simulation parameters.

\begin{tabular}{|c|c|c|}
\hline Parameter & Description & Value \\
\hline \hline$f_{c}$ & Carrier frequency & $2 \mathrm{GHz}$ \\
\hline$P_{i}$ & UAV transmit power & $0.5 \mathrm{~W}$ \\
\hline$N_{o}$ & Noise power spectral & $-170 \mathrm{dBm} / \mathrm{Hz}$ \\
\hline$N$ & Number of ground users & 300 \\
\hline$\mu_{\mathrm{LoS}}$ & Additional path loss to free space for LoS & $3 \mathrm{~dB}$ \\
\hline$\mu_{\mathrm{NLOS}}$ & Additional path loss to free space for NLoS & $23 \mathrm{~dB}$ \\
\hline$B$ & Bandwidth & $1 \mathrm{MHz}$ \\
\hline$\alpha$ & Control time factor & 0.01 \\
\hline$h$ & UAV's altitude & $200 \mathrm{~m}$ \\
\hline$\mu_{x}, \mu_{y}$ & Mean of the truncated Gaussian distribution & $250 \mathrm{~m}, 330 \mathrm{~m}$ \\
\hline$b_{1}, b_{2}$ & Environmental parameters (dense urban) & $0.36,0.21[6]$ \\
\hline
\end{tabular}

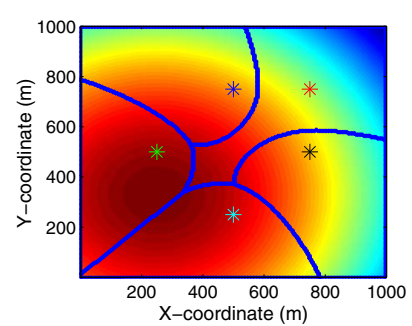

(a) Proposed optimal cell partitions.

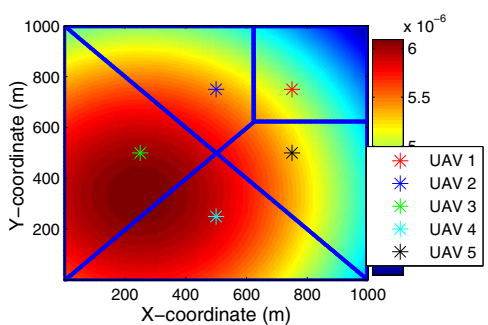

(b) Weighted Voronoi diagram.
Fig. 2: Cell partitions associated to UAVs given the non-uniform spatial distribution of users.

according to a two-dimensional truncated Gaussian distribution which is suitable to model a hotspot area [10]. Note that, although we consider the truncated Gaussian distribution of users, our analysis can also accommodate any other arbitrary distribution. We deploy the UAVs based on a grid-based deployment with an altitude of $200 \mathrm{~m}$. Unless stated otherwise, we consider a full interference scenario with an interference factor $\beta=1$. For the control time function, we consider $g_{i}(z)=\alpha z^{2}$, with $\alpha$ being an arbitrary constant factor. This function is a reasonable choice in our model as it is a superlinear function of the number of users and its value can be adjusted by factor $\alpha$. However, any arbitrary Lipschitz continuous control function can also be considered in our model. The simulation parameters are listed in Table I. We compare our results, obtained based on the proposed optimal cell partitioning approach, with the classical weighted Voronoi diagram baseline. Note that, all statistical results are averaged over a large number of independent runs.

Fig. 2 shows the proposed optimal cell partitions and the classical weighted Voronoi diagram. In this case, we consider 5 UAVs that provide service for the non-uniformly distributed ground users (truncated Gaussian distribution). Moreover, we assume that the maximum flight time of each UAV is 30 minutes which corresponds to the typical flight time for quadcopter UAVs. In Fig. 2, areas shown by a darker color have a higher population density. As we can see from Fig. $2 b$, the cell partitions associated with UAVs 3 and 4 have significantly more users than cell partition 1 . Therefore, given the limited flight times, users located at cell partitions 3 and 4 cannot be fairly served by UAVs. However, in the proposed optimal cell partitioning case (obtained by Algorithm 1), the cell partitions change such that the average data service under a fair resource allocation constraint is maximized. For instance, as shown in Fig. 2a, the size of cell partitions 3 and 4 decreases compared 


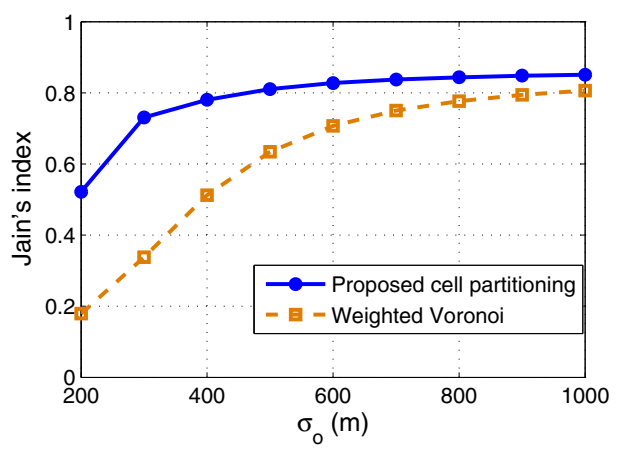

Fig. 3: Jain's fairness index for average data service to users.

to the weighted Voronoi diagram. As a result, the proposed cell partitions lead to a higher level of fairness among the users than the weighted Voronoi case.

To show how fairly the users can be served in different cases of cell partitioning, we use the Jain's fairness index. We compute the Jain's index based on the data service that is offered to each user which is given by [14]:

$$
F_{\text {Jain }}\left(l_{1}, l_{2}, \ldots, l_{N}\right)=\left(\sum_{i=1}^{N} l_{i}\right)^{2} \times\left(N \sum_{i=1}^{N} l_{i}^{2}\right)^{-1},
$$

where $N$ is the number of users, and $l_{i}$ is the data service to user $i$. Clearly, $1 / N \leq F_{\text {Jain }} \leq 1$, with $F_{\text {Jain }}=1 / N$ and $F_{\text {Jain }}=1$ indicating the lowest and highest level of fairness.

Fig. 3 shows the Jain's fairness index for different values of the standard deviation in the truncated Gaussian distribution, $\sigma_{o}$. In this figure, as $\sigma_{o}$ increases, the spatial distribution of users becomes closer to a uniform distribution. As we can see from this figure, the minimum Jain's index corresponding to the proposed cell partitioning method is above 0.5 . However, in the weighted Voronoi case, it can decrease to 0.18 for a highly non-uniform distribution of users with $\sigma_{o}=200 \mathrm{~m}$. This is due to the fact that, in the Voronoi case, users located in highly congested partitions receive lower service than the partitions with low number of users. In the proposed approach, however, the resources (flight time and bandwidth) are fairly shared between the users thus leading to a higher fairness index. From Fig. 3 we can also observe that, for higher values of $\sigma_{o}$ (more uniform distribution), the fairness index for the proposed approach becomes closer to the weighted Voronoi case.

Fig. 4 shows the average total data service as a function of the interference factor, $\beta$ used in (4). Clearly, as the interference between UAVs decreases, the total data service that they can provide to the ground users increases. For instance, by decreasing $\beta$ from 1 (full interference case) to 0.1 , the total data service increases by a factor of 3 when 5 UAVs are deployed. Fig. 4 also shows that the service gain achieved by using a higher number of UAVs is significant only when the interference between the UAVs is highly mitigated (low values of $\beta$ ). For example, increasing the number of UAVs from 5 to 10 can lead to $56 \%$ data service gain for $\beta=0.1$, while this gain is only $5 \%$ in the full interference case.

\section{CONCLUSION}

In this paper, we have proposed a novel framework for optimizing UAV-enabled wireless networks while taking into

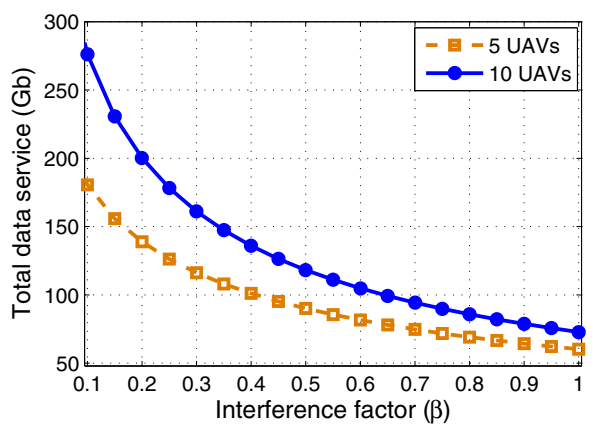

Fig. 4: Average data service versus interference factor.

account the flight time constraints of UAVs. In particular, given the maximum possible flight times of UAVs, we have maximized the average data service to the ground users under a fair resource allocation policy. To this end, using tools from optimal transport theory, we have determined the optimal cell partitions associated with the UAVs. The results have shown that, using our proposed cell partitioning approach, the users receive more fair data service compared to the Voronoi case.

\section{REFERENCES}

[1] M. Mozaffari, W. Saad, M. Bennis, and M. Debbah, "Unmanned aerial vehicle with underlaid device-to-device communications: Performance and tradeoffs," IEEE Transactions on Wireless Communications, vol. 15, no. 6, pp. 3949-3963, June 2016.

[2] A. Merwaday and I. Guvenc, "UAV assisted heterogeneous networks for public safety communications," in Proc. of IEEE Wireless Communications and Networking Conference Workshops (WCNCW), March 2015.

[3] M. Mozaffari, W. Saad, M. Bennis, and M. Debbah, "Mobile unmanned aerial vehicles (UAVs) for energy-efficient internet of things communications," available online: arxiv.org/abs/1703.05401, 2017.

[4] M. M. Azari, F. Rosas, K. C. Chen, and S. Pollin, "Joint sum-rate and power gain analysis of an aerial base station," in Proc. of IEEE Global Communications Conference (GLOBECOM) Workshops, Dec. 2016.

[5] I. Bor-Yaliniz and H. Yanikomeroglu, "The new frontier in RAN heterogeneity: Multi-tier drone-cells,' IEEE Communications Magazine, vol. 54, no. 11, pp. 48-55, 2016.

[6] A. Hourani, S. Kandeepan, and A. Jamalipour, "Modeling air-to-ground path loss for low altitude platforms in urban environments," in Proc. of IEEE Global Communications Conference (GLOBECOM), Austin, TX, USA, Dec. 2014.

[7] Y. Zeng and R. Zhang, "Energy-efficient UAV communication with trajectory optimization,' IEEE Transactions on Wireless Communications, vol. 16, no. 6, pp. 3747-3760, June 2017.

[8] Q. Wu, Y. Zeng, and R. Zhang, "Joint trajectory and communication design for UAV-enabled multiple access," available online: https://arxiv.org/abs/1704.01765., 2017.

[9] V. Sharma, M. Bennis, and R. Kumar, "UAV-assisted heterogeneous networks for capacity enhancement," IEEE Communications Letters, vol. 20, no. 6, pp. 1207-1210, June 2016.

[10] M. Mozaffari, W. Saad, M. Bennis, and M. Debbah, "Optimal transport theory for cell association in UAV-enabled cellular networks," IEEE Communications Letters, to appear, 2017.

[11] S. Niu, J. Zhang, F. Zhang, and H. Li, "A method of UAVs route optimization based on the structure of the highway network," International Journal of Distributed Sensor Networks, vol. 47, no. 1, pp. 70-85, 2015.

[12] C. Villani, Topics in optimal transportation. American Mathematical Soc., 2003, no. 58.

[13] G. Crippa, C. Jimenez, and A. Pratelli, "Optimum and equilibrium in a transport problem with queue penalization effect," Advances in Calculus of Variations, vol. 2, no. 3, pp. 207-246, 2009.

[14] R. Jain, D.-M. Chiu, and W. R. Hawe, A quantitative measure of fairness and discrimination for resource allocation in shared computer system. tech. rep., Digital Equipment Corporation, DEC-TR-301, 1984, vol. 38. 